\title{
openheart Prognostic value of left ventricular mitral annular longitudinal displacement obtained by tissue Doppler imaging in patients with heart failure with reduced ejection fraction
}

\author{
Morten Sengeløv (D) , ${ }^{1}$ Peter Godsk Jørgensen, ${ }^{1}$ Niels Eske Bruun, ${ }^{2}$ \\ Flemming Javier Olsen (D) , ${ }^{1}$ Thomas Fritz-Hansen, ${ }^{1}$ Tor Biering-Sørensen ${ }^{1}$
}

To cite: Sengeløv M, Jørgensen PG, Bruun NE, et al. Prognostic value of left ventricular mitral annular longitudinal displacement obtained by tissue Doppler imaging in patients with heart failure with reduced ejection fraction. Open Heart 2021;8:e001494. doi:10.1136/ openhrt-2020-001494

Received 29 0ctober 2020 Revised 8 January 2021 Accepted 8 January 2021
Check for updates

(C) Author(s) (or their employer(s)) 2021. Re-use permitted under CC BY-NC. No commercial re-use. See rights and permissions. Published by BMJ.

${ }^{1}$ Department of Cardiology, Herlev \& Gentofte Hospital, University of Copenhagen, Copenhagen, Denmark 2Department of Cardiology, Zealand University Hospital, University of Copenhagen, Copenhagen, Denmark

Correspondence to Dr Morten Sengeløv; mortensengelov@gmail.com

\section{ABSTRACT}

Background Tissue Doppler imaging (TDI) can be used to measure the mitral annular longitudinal displacement (LD) during systole. However, the prognostic utility of global and regional $L D$ in patients with heart failure with reduced ejection fraction (HFrEF) is unknown.

Methods Echocardiographic examinations from 907 patients with HFrEF were analysed obtaining conventional echocardiographic measurements. Regional LD was obtained from colour TDI projections in six mitral annular regions and global LD was calculated as an average.

Results Mean age was 67 years, $26.9 \%$ were women and mean left ventricular ejection fraction was $27 \%$. During a median follow-up period of 40 months, $150(16.5 \%)$ patients died. The risk of dying increased with decreasing tertile of global LD and was approximately five times higher for patients in the lowest tertile compared with the highest (1. tertile vs 3. tertile, HR 4.9, 95\% Cl: 3.0 to 7.9 , $\mathrm{p}<0.001)$.

Global LD was a significant independent predictor of mortality after adjusting for age, gender, body mass index, pacemaker, heart rate, atrial fibrillation, diabetes and conventional echocardiographic measures and global longitudinal strain: HR 1.16 (95\% Cl: 1.00 to 1.34 , $\mathrm{p}=0.044$ ) per $1 \mathrm{~mm}$ decrease.

For regional measures, inferior LD was also a significant independent predictor in the multivariable model: HR 1.16 (95\% Cl: 1.04 to $1.29, \mathrm{p}=0.006$ ) and adding inferior $\mathrm{LD}$ to the conventional measures yielded a significant increase in Harrell's C-statistic ( $95 \%$ Cl: 0.75 to $0.78, p=0.009)$. Conclusion In patients with HFrEF, global and inferior LD are independent predictors of all-cause mortality. Furthermore, inferior LD proved to be a significant prognosticator when compared with all the conventional echocardiographic parameters.

\section{INTRODUCTION}

Left ventricular (LV) annular longitudinal displacement (LD), obtained by colourcoded tissue Doppler imaging (TDI), may provide important prognostic information in patients with heart failure with reduced

\section{Key questions}

What is already known about this subject?

- Longitudinal displacement (LD) of the annular systolic plane has previously been shown to be a marker left ventricular systolic function.

What does this study add?

- Global LD obtained by tissue Doppler in heart failure with reduced ejection fraction (HFrEF) is significant predictor of mortality.

- Regional cardiac wall measures of LD provide improved diagnostics. An impaired inferior region of the left ventricle is particularly detrimental.

How might this impact on clinical practice?

- The findings allow for a diversified assessment of systolic function in HFrEF.

- Prognostic information in patients with poor image quality is obtainable through global and regional LD.

ejection fraction (HFrEF). The measurement is analogous to the mitral annular systolic plane excursion (MAPSE) obtained by M-mode. Beyond M-mode, the LD of the atrioventricular plane can be measured by various echocardiographic modalities including two-dimensional speckle tracking (2STE) and tissue tracking by TDI. ${ }^{1}$ Interestingly, TDI has recently been demonstrated to be the most robust technique to measure the LD when assessing the reproducibility, where it was found to have an interobserver variability (coefficient of variation) of $10 \%$ compared with $18 \%$ and $25 \%$ for 2STE and M-mode, respectively. ${ }^{2}$ In addition, the mean intraobserver variability was shown to be $-0.1 \mathrm{~mm}$ (SD: $\pm 1.8 \mathrm{~mm}$ ) and mean interobserver variability was shown to be $-0.1 \mathrm{~mm}$ (SD: $\pm 1.87 \mathrm{~mm})$. 
The LD by M-mode (MAPSE) is a well-established echocardiographic measurement. It has previously been shown to be a surrogate measure of left ventricular ejection fraction (LVEF), and it has been proven to be reduced in patients with HFrEF. ${ }^{34}$ In addition, lower LD has been demonstrated to be related to impaired diastolic function. ${ }^{5}$

In recent years, echocardiographic imaging techniques that quantify myocardial deformation, such as 2STE and TDI, have been subject to extensive research to determine prognostic capabilities beyond conventional echocardiographic measures. One measurement, global longitudinal strain (GLS), obtained by 2STE, has been of particular interest as it provides sensitive information about myocardial function and contractility. ${ }^{6}$ It has been proven to be a strong predictor of mortality in several patient populations including patients with $\mathrm{HFrEF}^{7}$ TDI has also been demonstrated to have an important role in the non-invasive estimation of LV filling pressure, ${ }^{8}$ and has been shown to be an important prognosticator of cardiac morbidity ${ }^{9-12}$ and in HFrEF. ${ }^{13}$ However, the prognostic value of $\mathrm{LD}$, measured by TDI, in patients with HFrEF has not previously been investigated. One advantage of measuring LD by TDI from the colourcoded projections is that only a relatively small region of the mitral plane needs to be visualised. In contrast, GLS by 2STE measurements require an adequate number of speckles sufficiently visualised from the entire LV which can become obscured in poor image quality. Therefore, if LD by TDI provides prognostic information comparable with GLS and LVEF, this method may be a fast and reproducible way to evaluate systolic function in the clinical setting.

Consequently, this study sought to elucidate if LD provides prognostic information from a clinical echocardiographic examination comparable with other echocardiographic measures routinely assessed in patients with HFrEF (especially LVEF and GLS). Furthermore, we sought to examine whether LDs obtained from the different LV regions confer differential or similar prognostic information.

\section{METHODS \\ Study population}

The cohort and patient selection process has previously been described in detail. ${ }^{7}$ Briefly, echocardiograms from an established heart failure clinic were retrieved and a total of 1065 patients with HFrEF had echocardiographic images eligible for analysis. For this study, 55 patients did not have any TDI recorded. In addition, 103 patients did not have colour-coded TDI measurements obtained from all six myocardial walls since they did not have colour-coded TDI from all three apical projections and were therefore excluded. This resulted in a total of 907 patients to be included in the final analysis.

Ischaemic origin was defined as patients who had a history of coronary artery bypass graft surgery, percutaneous transluminal coronary angioplasty or myocardial infarction.

We were able to retrieve data on coronary angiography (CAG) on a majority of the population (in 790 patients, $87 \%$ of the population) who had an elective CAG done. The procedure was done within a median of 39.5 days before examination (IQR: 412 days to 1 day before examination). A significant lesion was defined as having a stenosis of $70 \%$ or above in the right coronary artery (RCA), left anterior descending (LAD) artery, circumflex artery (CX) and left main stem (LMS).

\section{Echocardiography}

All clinical echocardiographic images were analysed by a blinded investigator (MS) for the purpose of this study. Information of the conventional echocardiographic measures and 2STE has previously been described in detail. ${ }^{7}$ LVEF was obtained using the modified Simpson's rule. ${ }^{14} \mathrm{LV}$ and diastolic dimensions were measured in the parasternal long axis view, and the anatomical LV mass was estimated by the Devereux formula. ${ }^{15}$ The left atrium volume was measured by the biplane area-length method, and tricuspid annular systolic plane excursion (TAPSE) was measured using M-mode echocardiography in the four-chamber apical view.

Pulsed-wave Doppler with the sample volume placed between the mitral leaflets in the four-chamber apical view was used to obtain the peak velocities of early and atrial diastolic LV filling and the deceleration time (DT) of the E-wave.

Regional LD was obtained in the apical four-chamber view by placing a sample volume in the septal and lateral annular sites. LD was then measured by tracking the tissue from the start of the systolic ejection period to the aortic valve closure (figure 1). Aortic valve opening and closure were measured by placing a curved M-mode through the mitral leaflet in the colour TDI. ${ }^{16}$ These steps were repeated for the two-chamber and three-chamber views in the remaining four annular sites (anterior, posterior, anterior septal and inferior). Global LD was calculated by averaging LD obtained from all six annular sites. Pulsedwave tissue Doppler in the four-chamber apical view was used to obtain septal and lateral peak longitudinal early diastolic (e') tissue velocity. The mean value was calculated as an average between the septal and the lateral velocities.

Two-dimensional speckle tracking and strain data were obtained in the apical two-chamber, three-chamber, fourchamber views and short axis view at the papillary muscle through semiautomated software.

In patients with atrial fibrillation (AF), the measures were obtained over one cardiac cycle similar to the rest of the population.

\section{Statistical analysis}

STATA statistics SE V.13.0 (StataCorp, Texas, USA) was used for computing the statistical analysis. For all analyses 


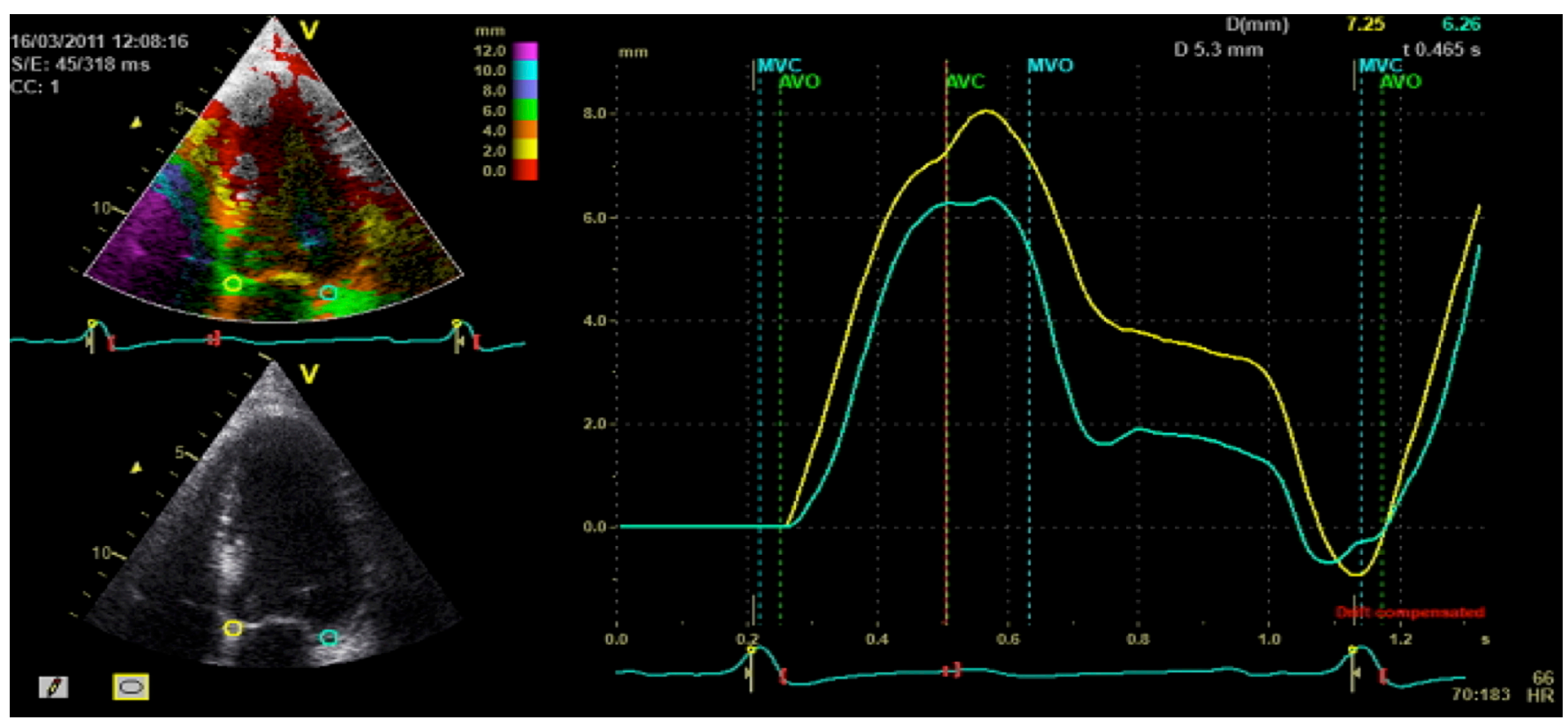

Figure 1 Measurement of mitral longitudinal displacement by tissue Doppler imaging. Regional LD is obtained in the apical four-chamber view by placing a sample volume in the septal and lateral annular sites. LD is then measured by tracking the tissue from the start of the systolic ejection period to the aortic valve closure. These steps are then repeated for the two-chamber and three-chamber in the remaining four annular sites (anterior, posterior, anterior septal and inferior). The measurement is expressed in millimetres. LD, longitudinal displacement.

performed, a $\mathrm{p}$ value in a two-sided test under 0.05 was considered statistically significant.

The clinical and echocardiographic variables were stratified based on tertiles of global LD, and $\mathrm{p}$ for trend across categories was tested. A survival curve based on global LD stratified into tertiles was constructed using the Kaplan-Meier method. Cox proportional hazards regression models were constructed, and both univariable and multivariable HRs were calculated. The assumption of proportional hazards was graphically asserted and tested based on the Schoenfeld residuals. In the multivariable regression models, we included age, gender, ischaemic origin and the variables that were significant predictors of mortality in the univariable Cox regression: body mass index (BMI), diabetes, mean arterial pressure (MAP), $\mathrm{AF}$, pacemaker (PM), heart rate, left ventricular internal dimension in diastole (LVIDd), LVEF, left ventricular mass index (LVMI), left atrial volume index (LAVI), E/e', TAPSE, DT and GLS. To quantify the prognostic strength of the examined parameters, Harrell's C-statistics were calculated from the univariable cox regression models, and multivariable models were compared with C-statistics by adding novel measures to models containing conventional echocardiographic and clinical parameters. Reclassification analysis was estimated through continuous net reclassification improvement (NRI).

\section{RESULTS}

\section{Global LD and mortality}

Of the 907 patients included, mean age was 67 years and $26.9 \%$ were women. During a median follow-up of 40 months (IQR: 58-22 months), 150 (16.5\%) patients died. Mean global LD for patients dead at follow-up was $5.01 \pm 2.41 \mathrm{~mm}$ compared with a mean global LD of $6.70 \pm 2.53 \mathrm{~mm}$ for patients alive at follow-up $(\mathrm{p}<0.001)$.

The patients' clinical characteristics and echocardiographic measures were stratified by tertiles of global LD (table 1). Lower global LD was associated with higher age and a higher prevalence of AF. For the echocardiographic measurements, patients with lower LD had larger LVMI, LVIDd, left ventricular end-diastolic volume and left ventricular end-systolic volume. Lower tertile of LD was associated with an impaired systolic function as seen by a lower LVEF, global circumferential strain, and impaired diastolic function as assessed by lower DT, average peak early diastolic mitral annular velocity (e') and higher peak transmitral early diastolic inflow velocity (E), E/e' and larger LAVI. Lastly, lower tertile of global LD was associated with an impaired right function as seen by a lower TAPSE.

\section{Global LD and prognostic value}

The patient population was stratified into tertiles of global LD. The risk of dying increased with decreasing tertile of LD and was approximately fivefold higher for patients in the lowest tertile compared with the highest (incidence rate: 1 . tertile $9.2,95 \%$ CI: 7.5 to 11.2 ; 2 . tertile 3.5, $95 \%$ CI: 2.5 to $4.8 ; 3$. tertile $1.9,95 \%$ CI: 1.2 to 2.9 ; Cox regression: 1 . tertile vs 3 . tertile, HR 4.9, 95\% CI: 3.0 to $7.9, \mathrm{p}<0.001$ ) (figure 2 ).

When assessed as a continuous variable, global LD was a significant predictor of mortality with a risk increase of $35 \%$ per $1 \mathrm{~mm}$ decrease in LD (HR 1.35 (95\% CI: 1.25 to 1.45$), \mathrm{p}<0.001$ ) (table 2 ). The variables that were 
Table 1 Clinical and echocardiographic parameters stratified by tertiles of global LD

\begin{tabular}{|c|c|c|c|c|c|}
\hline & Total & 1. tertile & 2. tertile & 3. tertile & P for trend across tertiles \\
\hline \multirow[t]{2}{*}{ Global LD (mm) } & $6.5 \pm 2.5$ & $3.8 \pm 1.0$ & $6.4 \pm 0.7$ & $9.2 \pm 1.4$ & \\
\hline & $\mathrm{n}=907$ & $\mathrm{n}=303$ & $\mathrm{n}=302$ & $\mathrm{n}=302$ & \\
\hline Event rate (per 100 patient-years, 95\% Cl) & & 9.2 (7.5 to 11.2$)$ & $3.5(2.5$ to 4.8$)$ & 1.9 (1.2 to 2.9$)$ & \\
\hline \multicolumn{6}{|l|}{ Clinical characteristic and comorbidities } \\
\hline Age (years) & $66.7 \pm 7.5$ & $68.5 \pm 10.2$ & $67.3 \pm 11.1$ & $64.3 \pm 12.7$ & $<0.001$ \\
\hline Male & $663(73.1 \%)$ & $226(74.6 \%)$ & $214(70.9 \%)$ & $223(73.8 \%)$ & 0.84 \\
\hline Heart rate (beats/min) & $73.6 \pm 15.5$ & $79.7 \pm 16.9$ & $72.3 \pm 14.9$ & $68.7 \pm 12.3$ & $<0.001$ \\
\hline $\mathrm{BMI}\left(\mathrm{kg} / \mathrm{m}^{2}\right)$ & $26.5 \pm 4.9$ & $25.8 \pm 4.6$ & $26.8 \pm 5.5$ & $26.9 \pm 4.2$ & 0.005 \\
\hline $\operatorname{MAP}(\mathrm{mm} \mathrm{Hg})$ & $92.9 \pm 13.4$ & $90.1 \pm 13.8$ & $92.9 \pm 13.4$ & $95.7 \pm 12.6$ & $<0.001$ \\
\hline AF & $140(15.4 \%)$ & $70(23.1 \%)$ & $47(15.6 \%)$ & $23(7.6 \%)$ & $<0.001$ \\
\hline Diabetes & $114(12.6 \%)$ & $39(12.9 \%)$ & $36(11.9 \%)$ & $39(12.9 \%)$ & 0.99 \\
\hline Ischaemic origin & $536(59.1 \%)$ & $172(56.8 \%)$ & $171(56.6 \%)$ & $193(63.9 \%)$ & 0.07 \\
\hline History of Ml & $439(48.4 \%)$ & $146(48.2 \%)$ & $140(46.4 \%)$ & $153(50.7 \%)$ & 0.54 \\
\hline PTCA & $261(28.8 \%)$ & $62(20.5 \%)$ & $83(27.5 \%)$ & $116(38.4 \%)$ & $<0.001$ \\
\hline CABG & $203(22.4 \%)$ & $69(22.8 \%)$ & $72(23.8 \%)$ & $62(20.5 \%)$ & 0.51 \\
\hline PM & $50(5.5 \%)$ & $27(8.9 \%)$ & $16(5.3 \%)$ & $7(2.3 \%)$ & 0.001 \\
\hline ICD & $25(2.8 \%)$ & $11(3.6 \%)$ & $9(3.0 \%)$ & $5(1.7 \%)$ & 0.14 \\
\hline Cholesterol (mmol/L) & $4.4 \pm 1.1$ & $4.4 \pm 1.1$ & $4.5 \pm 1.1$ & $4.4 \pm 1.2$ & 0.73 \\
\hline \multicolumn{6}{|l|}{ Echocardiographic parameters } \\
\hline LVIDd (cm) & $5.6 \pm 1.0$ & $6.1 \pm 1.0$ & $5.6 \pm 0.9$ & $5.3 \pm 0.9$ & $<0.001$ \\
\hline LVPWd (cm) & $1.0 \pm 0.2$ & $1.0 \pm 0.2$ & $1.0 \pm 0.2$ & $1.0 \pm 0.3$ & 0.69 \\
\hline LVESV (mL) & $66 \pm 31$ & $85.3 \pm 35.9$ & $60.8 \pm 24.7$ & $51.3 \pm 19.3$ & $<0.001$ \\
\hline LVEDV (mL) & $88 \pm 33$ & $104.5 \pm 38.6$ & $82.9 \pm 27.3$ & $77.2 \pm 24.1$ & $<0.001$ \\
\hline LVEF (\%) & $27 \pm 9$ & $20.0 \pm 7.6$ & $28.0 \pm 7.3$ & $34.3 \pm 7.1$ & $<0.001$ \\
\hline LVMI $\left(\mathrm{g} / \mathrm{m}^{2}\right)$ & $120 \pm 38$ & $134.6 \pm 41.5$ & $117.9 \pm 34.5$ & $107.8 \pm 33.2$ & $<0.001$ \\
\hline LAVI $\left(\mathrm{mL} / \mathrm{m}^{2}\right)$ & $31 \pm 14$ & $36.0 \pm 14.4$ & $30.6 \pm 12.5$ & $27.2 \pm 13.1$ & $<0.001$ \\
\hline TAPSE (cm) & $1.9 \pm 0.6$ & $1.6 \pm 0.5$ & $1.9 \pm 0.5$ & $2.1 \pm 0.5$ & $<0.001$ \\
\hline DT (ms) & $190 \pm 78$ & $160.8 \pm 68.9$ & $194.3 \pm 78.7$ & $212.9 \pm 76.2$ & $<0.001$ \\
\hline$E(\mathrm{~m} / \mathrm{s})$ & $0.8 \pm 0.3$ & $0.9 \pm 0.3$ & $0.8 \pm 0.3$ & $0.8 \pm 0.3$ & $<0.001$ \\
\hline $\mathrm{e}^{\prime}(\mathrm{cm} / \mathrm{s})$ & $6.9 \pm 2.5$ & $6.2 \pm 2.3$ & $6.5 \pm 2.4$ & $7.9 \pm 2.6$ & $<0.001$ \\
\hline$E / e^{\prime}$ & $13.1 \pm 6.0$ & $15.5 \pm 6.9$ & $13.4 \pm 5.5$ & $10.6 \pm 4.2$ & $<0.001$ \\
\hline GLS (\%) & $-9.6 \pm 3.4$ & $-6.7 \pm 2.1$ & $-9.5 \pm 2.4$ & $-12.5 \pm 2.7$ & $<0.001$ \\
\hline GCS (\%) & $-10.3 \pm 4.2$ & $-8.6 \pm 3.9$ & $-10.2 \pm 3.4$ & $-12.3 \pm 4.3$ & $<0.001$ \\
\hline Global SR $\left(\mathrm{s}^{-1}\right)$ & $-0.5 \pm 0.2$ & $-0.4 \pm 0.1$ & $-0.5 \pm 0.1$ & $-0.7 \pm 0.2$ & $<0.001$ \\
\hline $\mathrm{S}^{\prime}(\mathrm{cm} / \mathrm{s})$ & $3.9 \pm 1.4$ & $2.7 \pm 0.9$ & $3.9 \pm 0.8$ & $5.1 \pm 1.1$ & $<0.001$ \\
\hline Inferior LD (mm) & $7.0 \pm 3.0$ & $4.1 \pm 1.8$ & $6.9 \pm 1.5$ & $10.1 \pm 2.0$ & $<0.001$ \\
\hline Lateral LD (mm) & $7.2 \pm 3.0$ & $4.3 \pm 1.6$ & $7.2 \pm 1.7$ & $10.2 \pm 2.2$ & $<0.001$ \\
\hline Septal LD (mm) & $6.0 \pm 2.8$ & $3.3 \pm 1.5$ & $5.9 \pm 1.5$ & $8.8 \pm 2.0$ & $<0.001$ \\
\hline Posterior LD (mm) & $7.3 \pm 3.1$ & $4.3 \pm 1.6$ & $7.2 \pm 1.7$ & $10.4 \pm 2.3$ & $<0.001$ \\
\hline Anterior septal LD (mm) & $4.8 \pm 2.4$ & $2.8 \pm 1.4$ & $4.7 \pm 1.7$ & $6.9 \pm 2.1$ & $<0.001$ \\
\hline Anterior LD (mm) & $6.4 \pm 2.8$ & $4.0 \pm 1.6$ & $6.3 \pm 1.7$ & $9.0 \pm 2.2$ & $<0.001$ \\
\hline
\end{tabular}

AF, atrial fibrillation; BMI, body mass index; CABG, coronary artery bypass grafting; DT, deceleration time; e', average peak early diastolic mitral annular velocity; E, peak transmitral early diastolic inflow velocity; GCS, global circumferential strain; GLS, global longitudinal strain; ICD, implantable cardioverter defibrillator; LAVI, left atrial volume index; LD, longitudinal displacement; LVEDV, left ventricular end-diastolic volume; LVEF, left ventricular ejection fraction; LVESV, left ventricular end-systolic volume; LVIDd, left ventricular internal dimension in diastole; LVMI, left ventricular mass index; LVPWd, left ventricular posterior wall diameter at diastole; MAP, mean arterial pressure; MI, myocardial infarction; PM, pacemaker; PTCA, percutaneous transluminal coronary angioplasty; SR, strain rate; TAPSE, tricuspid annular plane systolic excursion. 


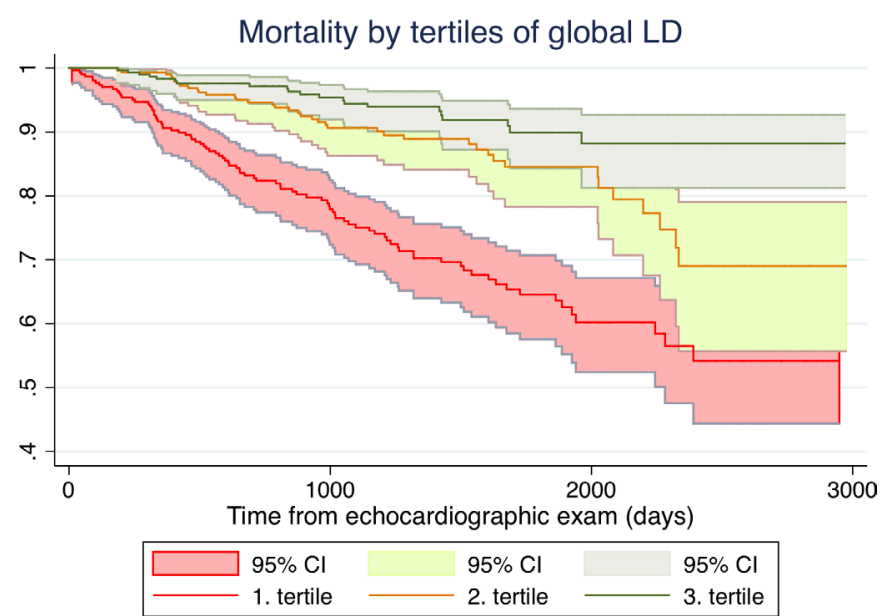

Figure 2 Kaplan-Meier curves for patients stratified into tertiles of LD. Depicting cumulative survival for the patient population stratified into tertiles of LD. LD, longitudinal displacement.

significant predictors of mortality in a univariable analysis are listed in table 2. In the multivariable models (including age, gender, ischaemic cardiomyopathy, BMI, diabetes, MAP, AF, PM, heart rate, LVIDd, LVEF, LVMI, LAVI, E/e', TAPSE, DT), global LD was the only significant echocardiographic predictor of mortality: HR 1.16 (95\% CI: 1.19 to $1.34, \mathrm{p}=0.006)$ per $1 \mathrm{~mm}$ decrease (table 3). Age, MAP, BMI and diabetes were significant clinical predictors of mortality in the multivariable analysis. Global LD remained significant even after adjusting for GLS: HR 1.16 (95\% CI: 1.00 to 1.34 , $\mathrm{p}=0.044$ ).

Global LD provided incremental prognostic information when added to LVEF as assessed by a significant NRI: 0.373 (95\% CI: 0.163 to 0.575 ). However, adding Global LD to all conventional predictors did not result in a significant NRI.

In a sensitivity analysis including all patients $(\mathrm{N}=1003)$ who had an LD measurement in any myocardial wall, thus not requiring that patients should have measurements available in all six myocardial walls, global LD remained a significant predictor of mortality in both univariable and multivariable models: HR 1.31 (95\% CI: 1.23 to 1.41, $\mathrm{p}<0.001)$ and HR 1.14 (95\% CI: 1.00 to $1.29, \mathrm{p}=0.044$ ).

\section{Prognostic utility of regional LD}

LD obtained from all six cardiac walls (inferior, lateral, septal, posterior, anterior septal, anterior) were significant predictors of mortality in the univariable Cox regression models $(p<0.001$ for all) (figure 3A) with LD obtained from the inferior wall having the highest C-statistics (0.72) of all the annular sites, but also compared with all other echocardiographic measurements (table 2). Figure 4 illustrates the relationship between the LD of each individual six annular site and incident mortality.

In the multivariable models including all the variables previously mentioned, LD obtained from four walls, respectively, remained significant predictors of mortality: lateral $(\mathrm{p}=0.036)$, septal $(\mathrm{p}=0.028)$, posterior $(\mathrm{p}=0.046)$ and inferior (0.001) (table 3 and figure 3B). After further adjustment for GLS, LD from the inferior wall remained a significant predictor of mortality: HR 1.16 (95\% CI: 1.04 to $1.29, \mathrm{p}=0.006$ ) (figure 3C). The other clinical predictors that remained significant were age, diabetes, MAP and BMI. Adding inferior LD to the clinical measurements and conventional echocardiographic predictors resulted in a better overall prediction model as seen by a significant increase in C-statistics from 0.75 to $0.78(\mathrm{p}=0.009)$ and a significant increase in NRI 0.356 (95\% CI: 0.079 to 0.550 ).

Of the 790 patients with CAG data available, $316(40 \%)$ did not have a significant lesion, 206 (26\%) patients had a single lesion and 268 (33\%) had two lesions or more. When including CAG data on significant coronary stenosis (RCA, LAD, CX and LMS) in the multivariable model, inferior LD remained a significant independent predictor of outcome: HR 1.19 (95\% CI: 1.07 to 1.33), $\mathrm{p}=0.001$, even after further adjusting for GLS: HR 1.18 (95\% CI: 1.05 to 1.32 ), $\mathrm{p}=0.004$.

When individually assessing other systolic predictors of mortality in the same multivariable model with inferior LD such as systolic global strain rate and s' velocity by TDI, inferior LD remained a predictor of mortality $(\mathrm{p}<0.001$ and $\mathrm{p}=0.002)$. Systolic global strain rate and $\mathrm{s}$ velocity were not significant in the multivariable model, respectively.

\section{DISCUSSION}

This study examines the prognostic value of LD in a large HFrEF cohort and compares the prognostic utility of this simple measure of longitudinal deformation with conventional and novel measures of cardiac structure and function. We demonstrated three major principal findings: first, lower global LD is a significant predictor of mortality in patients with HFrEF. Second, additional prognostic information is gained from regional LD from all six cardiac walls. Third, LD from the inferior myocardial wall provides prognostic information beyond conventional and novel echocardiographic measurements. In addition, significant incremental prognostic information is attained by including an assessment of inferior LD to the clinical and conventional echocardiographic measures.

The evidence of tissue tracking, by TDI, as a viable echocardiographic technique has been growing in recent years. A study determined that the LD correlated well with clinical markers of heart failure such as brain natriuretic peptide and New York Heart Association (NYHA)class. ${ }^{17}$ Another study found that LD predicted mortality in patients with non-ST-elevation myocardial infarction (STEMI) ${ }^{18}$ and LD has been proven to correlate well with myocardial infarct size. ${ }^{19}$ In addition, a study has determined that tissue tracking by TDI can be used to evaluate regional myocardial pathophysiology in the setting of cardiac resynchronisation therapy. ${ }^{20}$ 
Table 2 Univariable Cox proportional hazard models for clinical and echocardiographic variables

\begin{tabular}{|c|c|c|c|c|}
\hline \multirow[b]{2}{*}{ Variable } & \multicolumn{4}{|c|}{ Univariable analysis } \\
\hline & HR & $95 \% \mathrm{Cl}$ & C-statistic & $P$ value \\
\hline Age (per 1 year increase) & 1.04 & 1.03 to 1.06 & 0.63 & $<0.001$ \\
\hline Heart rate (per 1 beat/min increase) & 1.01 & 1.0 to 1.01 & 0.58 & 0.003 \\
\hline $\mathrm{AF}$ & 1.98 & 1.37 to 2.87 & 0.56 & $<0.001$ \\
\hline Diabetes & 1.92 & 1.29 to 2.87 & 0.53 & 0.001 \\
\hline PM & 2.15 & 1.24 to 3.72 & 0.52 & 0.007 \\
\hline BMI (per $1 \mathrm{~kg} / \mathrm{m}^{2}$ increase) & 0.96 & 0.92 to 0.99 & 0.57 & 0.017 \\
\hline MAP (per $1 \mathrm{~mm} \mathrm{Hg} \mathrm{increase)}$ & 0.97 & 0.95 to 0.98 & 0.65 & $<0.001$ \\
\hline Cholesterol (per $1 \mathrm{mmol} / \mathrm{L}$ increase) & 2.15 & 0.70 to 96 & 0.56 & 0.014 \\
\hline LVIDd (per $1 \mathrm{~cm}$ increase) & 1.20 & 1.03 to 1.42 & 0.57 & 0.021 \\
\hline LVEDV (per $1 \mathrm{~mL}$ increase) & 1.01 & 1.01 to 1.02 & 0.62 & $<0.001$ \\
\hline LVESV (per $1 \mathrm{~mL}$ increase) & 1.00 & 1.00 to 1.01 & 0.59 & $<0.001$ \\
\hline LVEF (per 1\% decrease) & 1.06 & 1.04 to 0.1 .08 & 0.66 & $<0.001$ \\
\hline LVMI (per $1 \mathrm{~g} / \mathrm{m}^{2}$ decrease) & 1.01 & 1.00 to 1.01 & 0.57 & 0.01 \\
\hline LAVI (per $\mathrm{mL} / \mathrm{m}^{2}$ decrease) & 1.03 & 1.01 to 1.03 & 0.63 & $<0.001$ \\
\hline TAPSE (per $1 \mathrm{~mm}$ increase) & 0.92 & 0.89 to 0.95 & 0.63 & $<0.001$ \\
\hline $\mathrm{E}$ (per $1 \mathrm{~m} / \mathrm{s}$ increase) & 1.95 & 1.18 to 3.32 & 0.58 & 0.009 \\
\hline DT (per 1 ms decrease) & 0.997 & 0.99 to 1.00 & 0.58 & 0.011 \\
\hline $\mathrm{e}^{\prime}$ (per $1 \mathrm{~cm} / \mathrm{s}$ increase) & 0.93 & 0.86 to 0.99 & 0.57 & 0.026 \\
\hline E/e' (per 1 decrease) & 1.04 & 1.02 to 1.07 & 0.62 & $<0.001$ \\
\hline GLS (per 1\% decrease) & 1.22 & 1.15 to 1.28 & 0.69 & $<0.001$ \\
\hline GCS (per 1\% decrease) & 1.12 & 1.06 to 1.17 & 0.64 & $<0.001$ \\
\hline Global SR (per s ${ }^{-1}$ decrease) & 16.4 & 6.3 to 42.5 & 0.66 & $<0.001$ \\
\hline $\mathrm{S}^{\prime}$ (per $1 \mathrm{~cm} / \mathrm{s}$ decrease) & 1.43 & 1.27 to 1.61 & 0.68 & $<0.001$ \\
\hline Global LD (per $1 \mathrm{~mm}$ decrease) & 1.35 & 1.25 to 1.45 & 0.71 & $<0.001$ \\
\hline Inferior LD (per $1 \mathrm{~mm}$ decrease) & 1.29 & 1.22 to 1.37 & 0.72 & $<0.001$ \\
\hline Lateral LD (per $1 \mathrm{~mm}$ decrease) & 1.19 & 1.12 to 1.26 & 0.66 & $<0.001$ \\
\hline Septal LD (per $1 \mathrm{~mm}$ decrease) & 1.29 & 1.20 to 1.36 & 0.70 & $<0.001$ \\
\hline Posterior LD (per $1 \mathrm{~mm}$ decrease) & 1.19 & 1.13 to 1.26 & 0.67 & $<0.001$ \\
\hline Anterior septal LD (per $1 \mathrm{~mm}$ decrease) & 1.30 & 1.20 to 1.41 & 0.66 & $<0.001$ \\
\hline Anterior LD (per $1 \mathrm{~mm}$ decrease) & 1.21 & 1.14 to 1.29 & 0.66 & $<0.001$ \\
\hline
\end{tabular}

AF, atrial fibrillation; BMI, body mass index; DT, deceleration time; e', average peak early diastolic mitral annular velocity; E, peak transmitral early diastolic inflow velocity; GCS, global circumferential strain; GLS, global longitudinal strain; LAVI, left atrial volume index; LD, longitudinal displacement; LVEF, left ventricular ejection fraction; LVESV, left ventricular end-systolic volume; LVIDd, left ventricular internal dimension in diastole; LVMI, left ventricular mass index; MAP, mean arterial pressure; PM, pacemaker; SR, strain rate; TAPSE, tricuspid annular plane systolic excursion.

Our study gives basis for the hypothesis that global LD is a systolic prognosticator comparable with LVEF and more importantly GLS, which are both significant predictors of mortality in patients with HFrEF. ${ }^{721}$ This is emphasised by the fact that global LD was a significant predictor of mortality in the multivariable model including conventional echocardiographic measures (table 3). The strong prognostic capabilities of global LD might be due to the fact that it is a measure which captures information about both systolic and diastolic function. Displacement of the mitral annulus towards the apex during systole stores potential energy within the myocardial tissue and wall which, like a spring, contributes to the early relaxation and filling of the LV during diastole. Hence, the magnitude of global LD during systole affects the diastolic relaxation and function. In support of this theory, a study has previously demonstrated that the movement of the atrioventricular plane is related to the diastolic function as assessed by Doppler measurements. ${ }^{5}$ This was also evident in our study where a lower LD was associated with both lower systolic performance and worse diastolic function across all Doppler measurements obtained. In addition, the predictive capability of LD by TDI in HFrEF may also be due to a high degree of precision as a result of a high 


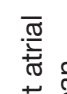

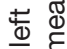

安定

至

잉

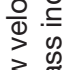

은

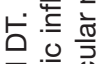

完

㟧 $\frac{\pi}{0}$

这亲电

(1) $\frac{0}{\overline{2}}$

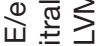

₹ $\frac{\varepsilon}{5}$

需

离

入

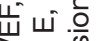

入

융응 reproducibility of the measure, which has been found to have an intraobserver and interobserver variability (coefficient of variation) of $11 \%$ and $10 \%$, respectively. ${ }^{2}$

In our analysis, LD measures obtained from every cardiac wall were significant predictors of mortality (table 2 and figure 3). When independently assessing LD obtained from every wall in a multivariable model including conventional echocardiographic measures, we observed that the anterior and anterior septal parts of the heart did not contribute with prognostic information. In the multivariable model, LD obtained from the inferior wall was the only significant echocardiographic predictor of mortality among all echocardiographic measures (figure 3). Thus, the predictive value of LD seems to be derived from the longitudinal deformation of the inferior wall. A similar pattern has been observed in a study where the myocardial velocity of the inferior wall, obtained by TDI, was better in predicting ventricular arrhythmias and cardiovascular mortality in patients with HFrEF. ${ }^{9}$ The authors hypothesised that the predictive capability could be explained by regionally increased wall-stress stemming from a greater radius of curvature of the LV's inferior wall. ${ }^{22}$ Another study found that e' of the inferior wall was the strongest diastolic predictor of mortality in patients with cerebral infarcts ${ }^{12}$ and it was recently presented that an impaired longitudinal function of the inferior wall, regardless if obtained by LD or 2STE, was the strongest deformation predictor (beyond GLS and LVEF) of adverse outcomes following a STEMI. ${ }^{23}$ Furthermore, in 1064 patients enrolled in the MADIT-CRT trial, longitudinal strain obtained from the inferior myocardial wall was superior for predicting ventricular arrhythmias. ${ }^{24}$ Having an impaired inferior wall appears to harvest a great risk of events across the spectra of cardiovascular diseases and echocardiographic modalities. Analyses from the CAG subgroup suggest that regional ischaemia does not influence the prognostic capability of LD. With these studies taken into consideration, there is growing evidence that incremental prognostic information can be gained by using regional analyses of the myocardium through novel imaging techniques such as 2STE and TDI.

In regard to the regional LV analysis in HFrEF, an aspect to consider is that these patients are affected by a neurohormonal imbalance through an increased activation of the sympathetic nervous system. ${ }^{25}$ However, myocardial scintigraphy has shown that sympathetic innervation of the inferior wall decreases with age ${ }^{26}$ and in diabetics. ${ }^{27}$ Thus, the inferior region of the heart may more adequately reflect the unstimulated intrinsic condition of the myocardial performance and be less influenced by humeral and autonomic regulations. This may explain why an impaired inferior wall may be a particular relevant area of the heart to evaluate when assessing risk in patients with HFrEF.

The results of our study suggest that LD obtained by TDI may have the potential to be a useful clinical systolic marker in patients with HFrEF. Global or regional LD could be easily implemented clinically due to the simple 
A)

Mean regional LD (SD) in non-event versus events and univariable HR per $1 \mathrm{~mm}$ decrease

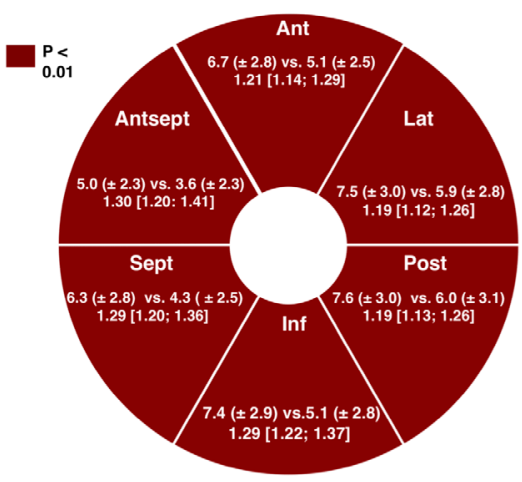

B)

HR per $1 \mathrm{~mm}$ decrease for regional $L D$ in a multivariable model including conventional measures

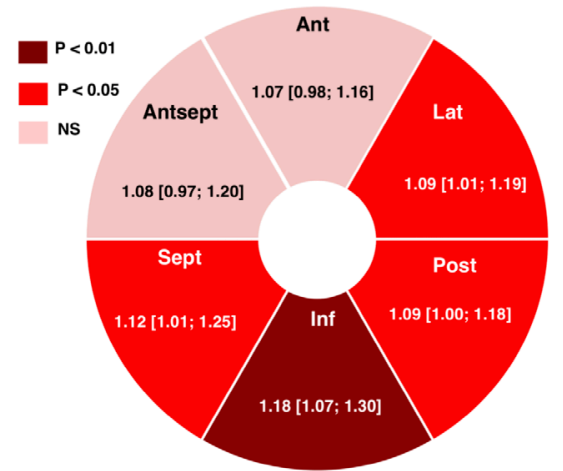

C)

HR per $1 \mathrm{~mm}$ decrease for regional LD in a multivariable model including conventional measures and GLS

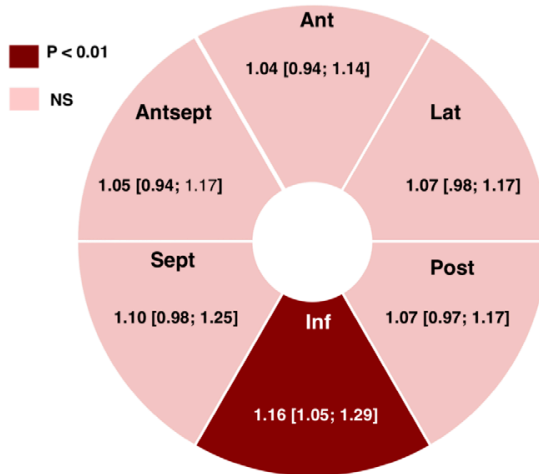

Figure 3 Regional LD and prognosis. (A) Regional LD (mean \pm SD) for patients alive or dead at follow-up, and univariable HRs describing the risk of mortality per $1 \mathrm{~mm}$ decrease in LD for each myocardial wall. (B) HR per $1 \mathrm{~mm}$ decrease in LD obtained from each myocardial wall. The multivariable model includes age, gender, ischaemic origin, BMI, diabetes, MAP, AF, PM, heart rate, LVIDd, LVEF, LVMI, LAVI, E velocity, E', E/e', TAPSE and DT. (C) HR per $1 \mathrm{~mm}$ decrease in LD obtained from each myocardial wall. The multivariable model includes age, gender, ischaemic origin, BMI, diabetes, MAP, AF, heart rate, LVIDd, LVEF, LVMI, LAVI, E velocity, TAPSE, DT and GLS. AF, atrial fibrillation; BMI, body mass index; DT, deceleration time; E, peak transmitral early diastolic inflow velocity; e', average peak early diastolic mitral annular velocity; GLS, global longitudinal strain; LAVI, left atrial volume index; LD, longitudinal displacement, LVEF, left ventricular ejection fraction; LVIDd, left ventricular internal dimension in diastole; LVMI, left ventricular mass index; PM, pacemaker; TAPSE, tricuspid annular plane systolic excursion.
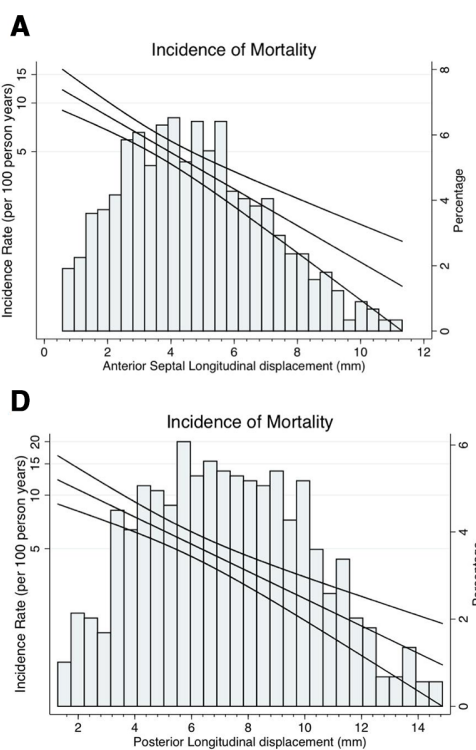

B

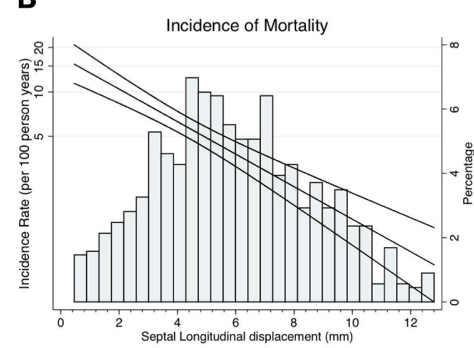

E

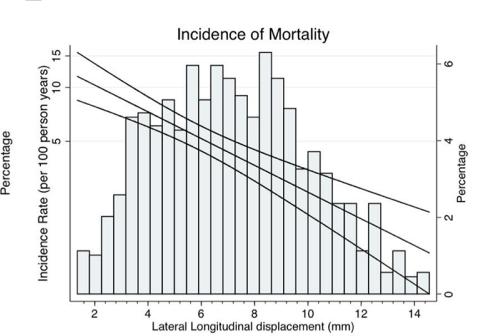

C
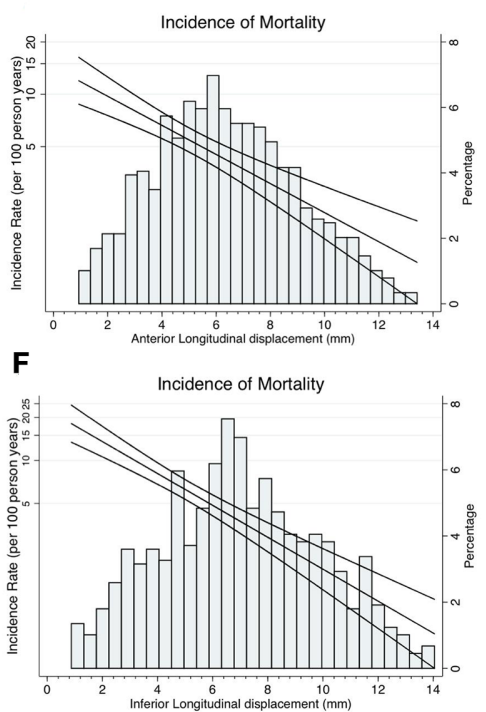

Figure 4 Incidence rates of mortality based on LD obtained from each myocardial wall. (A) Association between anterior septal LD and mortality. The incident of death per 100 patient-years is depicted on the $y$-axis and LD in millimetre on the $x$-axis. The graph is superimposed on a histogram of anterior septal LD of the HFrEF cohort. (B) Association between septal LD and mortality. The incident of death per 100 patient-years is depicted on the $y$-axis and LD in millimetre on the x-axis. The graph is superimposed on a histogram of septal LD of the HFrEF cohort. (C) Association between anterior LD and mortality. The incident of death per 100 patient-years is depicted on the $y$-axis and LD in millimetre on the $x$-axis. The graph is superimposed on a histogram of anterior LD of the HFrEF cohort. (D) Association between posterior LD and mortality. The incident of death per 100 patient-years is depicted on the $y$-axis and LD in millimetre on the $x$-axis. The graph is superimposed on a histogram of posterior LD of the HFrEF cohort. (E) Association between lateral LD and mortality. The incident of death per 100 patientyears is depicted on the $y$-axis and LD in millimetre on the x-axis. The graph is superimposed on a histogram of lateral LD. (F) Association between inferior LD and mortality. The incident of death per 100 patient-years is depicted on the $y$-axis and LD in millimetre on the x-axis. The graph is superimposed on a histogram of inferior LD. HFrEF, heart failure with reduced ejection fraction; LD, longitudinal displacement. 
and quick method in which the measurement is obtained (figure 1). In addition, it is intuitively expressed in millimetres and the TDI method provides a colour-coded scheme where the clinician can readily observe depressed regionally myocardial function. However, we do not suggest the measurements should replace existing and emerging predictors of mortality in LV dysfunction but rather complement and diversify the echocardiographic approach of evaluating systolic function.

Indeed, an instance where LD could be of particular clinical value is in the case of poor image quality of the LV. Strain analyses require optimal visualisation of the whole myocardium, and LVEF delineation necessitates good visualisation of the endocardium in both the apical four-chamber and two-chamber views. LD can be used with a limited acoustic window ${ }^{19}$ in the three apical views when only the basal regions are visualised and available for analyses. Consequently, it would be favourable in a clinical setting to have an echocardiographic measurement that could supplement the conventional measures and GLS in the case of poor image quality. Additionally, we observed that LD obtained from the inferior wall was the single best regional cardiac deformation measure to identify patients in high risk of mortality. It might be valuable in the clinical setting to be able to quickly gain accurate prognostic from a small area when assessing risk of mortality in HFrEF.

\section{Limitations}

Important limitations have to be taken into account for this analysis. Although we accounted for cardiovascular diseases found in patients with HFrEF, we cannot eliminate the possibility that other comorbidities such as cancer, chronic obstructive pulmonary disease or hepatic cirrhosis, which influences the prognosis of mortality, ${ }^{28}$ were present and influenced our estimations in the multivariable models. Furthermore, we did not have sufficient data on NYHA-class, which has demonstrated to be of important clinical significance in patients with HFrEF. ${ }^{29} 30$ However, mitral LD has been shown to correlate well with NYHA-class. ${ }^{17}$ We did not have outcome data beyond all-cause mortality; however, an assessment of specific cardiovascular mortality would most likely strengthen the prognostic capability of the LD measures in the cohort.

\section{CONCLUSION}

Global LD is a prognosticator in patients with HFrEF comparable with other established systolic prognosticators such as GLS and LVEF. In addition, LD obtained from the inferior wall is an independent predictor of allcause mortality beyond conventional and novel echocardiographic measurements.

Acknowledgements The authors wish to thank the staff and participants of the study for their important contributions.

Contributors MS, TB-S and PGJ made the study design and concept. All authors have participated in analysing and interpretation of the data. All authors revised the paper. All authors have approved this paper in its final submitted form.
Funding Work for this manuscript was supported by a research grant from the Novo Nordisk Research Foundation (grant number NNF150C0017456).

Competing interests PGJ has received lecture fees from Novo Nordisk and AstraZeneca. TB-S reports receiving research grants from Sanofi Pasteur and GE Healthcare, being a Steering Committee member of the Amgen financed GALACTIC-HF trial, on advisory boards for Sanofi Pasteur and Amgen, and speaker honorariums from Novartis and Sanofi Pasteur.

Patient consent for publication Not required.

Ethics approval The study complied with the declaration of Helsinki. The study was approved by the Danish Data Protection Agency, journal no. 03240 (I-Suite), ID: GEH-2014-047. In Denmark, retrospective studies do not need further approval from the local scientific ethics committee.

Provenance and peer review Not commissioned; externally peer reviewed.

Data availability statement Data may be obtained from a third party upon request but are not publicly available.

Open access This is an open access article distributed in accordance with the Creative Commons Attribution Non Commercial (CC BY-NC 4.0) license, which permits others to distribute, remix, adapt, build upon this work non-commercially, and license their derivative works on different terms, provided the original work is properly cited, appropriate credit is given, any changes made indicated, and the use is non-commercial. See: http://creativecommons.org/licenses/by-nc/4.0/.

Correction notice This article has been corrected since first published online. Author name 'Peter Godsk' has been corrected to 'Peter Godsk Jørgensen'.

ORCID iDs

Morten Sengeløv http://orcid.org/0000-0002-2139-8068

Flemming Javier Olsen http://orcid.org/0000-0001-9511-8375

\section{REFERENCES}

1 Pan $\mathrm{C}$, Hoffmann $\mathrm{R}$, Kühl $\mathrm{H}$, et al. Tissue tracking allows rapid and accurate visual evaluation of left ventricular function. Eur $J$ Echocardiogr 2001;2:197-202.

2 de Knegt MC, Biering-Sørensen T, Sogaard P, et al. Concordance and reproducibility between $\mathrm{M}$-mode, tissue Doppler imaging, and two-dimensional strain imaging in the assessment of mitral annular displacement and velocity in patients with various heart conditions. Eur Heart J Cardiovasc Imaging 2014;15:62-9.

3 Matos J, Kronzon I, Panagopoulos G, et al. Mitral annular plane systolic excursion as a surrogate for left ventricular ejection fraction. $J$ Am Soc Echocardiogr 2012;25:969-74.

4 Alam M, Höglund C, Thorstrand C, et al. Atrioventricular plane displacement in severe congestive heart failure following dilated cardiomyopathy or myocardial infarction. J Intern Med 1990;228:569-75.

5 Willenheimer R, Israelsson B, Cline C. Left atrioventricular plane displacement is related to both systolic and diastolic left ventricular performance in patients with chronic heart failure. Eur Heart $J$ 1999;20:612-8.

6 Perk G, Tunick PA, Kronzon I. Non-Doppler two-dimensional strain imaging by echocardiography--from technical considerations to clinical applications. J Am Soc Echocardiogr 2007;20:234-43.

7 Sengeløv M, Jørgensen PG, Jensen JS, et al. Global longitudinal strain is a superior predictor of all-cause mortality in heart failure with reduced ejection fraction. JACC Cardiovasc Imaging 2015;8:1351-9.

8 Nagueh SF, Middleton KJ, Kopelen HA, et al. Doppler tissue imaging: a noninvasive technique for evaluation of left ventricular relaxation and estimation of filling pressures. J Am Coll Cardiol 1997;30:1527-33.

9 Biering-Sørensen T, Olsen FJ, Storm K, et al. Prognostic value of tissue Doppler imaging for predicting ventricular arrhythmias and cardiovascular mortality in ischaemic cardiomyopathy. Eur Heart $J$ Cardiovasc Imaging 2016;17:722-31.

10 Mogelvang R, Biering-Sørensen T, Jensen JS. Tissue Doppler echocardiography predicts acute myocardial infarction, heart failure, and cardiovascular death in the general population. Eur Heart $J$ Cardiovasc Imaging 2015;16:jev180.

11 Biering-Sørensen T, Jensen JS, Pedersen S, et al. Doppler tissue imaging is an independent predictor of outcome in patients with ST-segment elevation myocardial infarction treated with primary percutaneous coronary intervention. J Am Soc Echocardiogr 2014;27:258-67. 
12 Olsen FJ, Jørgensen PG, Møgelvang R, et al. Diastolic myocardial dysfunction by tissue Doppler imaging predicts mortality in patients with cerebral infarction. Int $J$ Cardiovasc Imaging 2015;31:1413-22

13 Hamdan A, Shapira Y, Bengal T, et al. Tissue Doppler imaging in patients with advanced heart failure: relation to functional class and prognosis. J Heart Lung Transplant 2006;25:214-8.

14 Lang RM, Badano LP, Mor-Avi V, et al. Recommendations for cardiac chamber quantification by echocardiography in adults: an update from the American Society of echocardiography and the European association of cardiovascular imaging. J Am Soc Echocardiogr 2015;28:1-39.

15 Devereux RB, Reichek N. Echocardiographic determination of left ventricular mass in man. anatomic validation of the method. Circulation 1977:55:613-8.

16 Biering-Sørensen T, Mogelvang R, de Knegt MC, et al. Cardiac time intervals by tissue Doppler imaging M-Mode: normal values and association with established echocardiographic and invasive measures of systolic and diastolic function. PLoS One 2016;11:e0153636.

17 Ito K, Noma M, Mohri M, et al. Mitral annulus displacement measured by tissue-tracking method with Doppler-tissue images is a useful marker of the severity of heart failure. $J$ Cardiol 2007;50:159-66.

18 Zahid W, Johnson J, Westholm C, et al. Mitral annular displacement by Doppler tissue imaging may identify coronary occlusion and predict mortality in patients with non-ST-elevation myocardial infarction. J Am Soc Echocardiogr 2013;26:875-84.

19 Gjesdal O, Vartdal T, Hopp E, et al. Left ventricle longitudinal deformation assessment by mitral annulus displacement or global longitudinal strain in chronic ischemic heart disease: are they interchangeable? J Am Soc Echocardiogr 2009;22:823-30.

20 Søgaard P, Egeblad H, Kim WY, et al. Tissue Doppler imaging predicts improved systolic performance and reversed left ventricular remodeling during long-term cardiac resynchronization therapy. $J$ Am Coll Cardiol 2002;40:723-30.
21 Curtis JP, Sokol SI, Wang Y, et al. The association of left ventricular ejection fraction, mortality, and cause of death in stable outpatients with heart failure. J Am Coll Cardiol 2003;42:736-42.

22 Kuecherer HF, Kee LL, Modin G, et al. Echocardiography in serial evaluation of left ventricular systolic and diastolic function: importance of image acquisition, quantitation, and physiologic variability in clinical and investigational applications. J Am Soc Echocardiogr 1991;4:203-14.

23 Biering-Sørensen T, Jensen JS, Pedersen SH, et al. Regional longitudinal myocardial deformation provides incremental prognostic information in patients with ST-segment elevation myocardial infarction. PLoS One 2016;11:e0158280.

24 Biering-Sørensen T, Knappe D, Pouleur A-C, et al. Regional longitudinal deformation improves prediction of ventricular tachyarrhythmias in patients with heart failure with reduced ejection fraction: a MADIT-CRT substudy (multicenter automatic defibrillator implantation Trial-Cardiac resynchronization therapy). Circ Cardiovasc Imaging 2017;10:e005096.

25 Kaye DM, Lefkovits J, Jennings GL, et al. Adverse consequences of high sympathetic nervous activity in the failing human heart. $J \mathrm{Am}$ Coll Cardiol 1995;26:1257-63.

26 Sakata K, Shirotani M, Yoshida H, et al. Physiological fluctuation of the human left ventricle sympathetic nervous system assessed by iodine-123-MIBG. J Nucl Med 1998;39:1667-71.

27 Hattori N, Tamaki N, Hayashi T, et al. Regional abnormality of iodine123-MIBG in diabetic hearts. J Nucl Med 1996;37:1985-90.

28 Lee DS, Austin PC, Rouleau JL, et al. Predicting mortality among patients hospitalized for heart failure: derivation and validation of a clinical model. JAMA 2003;290:2581.

29 Madsen BK, Hansen JF, Stokholm KH, et al. Chronic congestive heart failure. description and survival of 190 consecutive patients with a diagnosis of chronic congestive heart failure based on clinical signs and symptoms. Eur Heart J 1994;15:303-10.

30 Muntwyler J, Abetel G, Gruner C, et al. One-Year mortality among unselected outpatients with heart failure. Eur Heart $J$ 2002;23:1861-6. 
Correction: Prognostic value of left ventricular mitral annular longitudinal displacement obtained by tissue doppler imaging in patients with heart failure with reduced ejection fraction

Sengeløv M, Jørgensen PG, Bruun NE, et al. Prognostic value of left ventricular mitral annular longitudinal displacement obtained by tissue Doppler imaging in patients with heart failure with reduced ejection fraction. Open Heart 2021;8:e001494. doi: 10.1136/ openhrt-2020-001494

Author name 'Peter Godsk' has been corrected to 'Peter Godsk Jørgensen'.

Open access This is an open access article distributed in accordance with the Creative Commons Attribution Non Commercial (CC BY-NC 4.0) license, which permits others to distribute, remix, adapt, build upon this work non-commercially, and license their derivative works on different terms, provided the original work is properly cited, appropriate credit is given, any changes made indicated, and the use is non-commercial. See: http://creativecommons.org/licenses/by-nc/4.0/.

C Author(s) (or their employer(s)) 2021. Re-use permitted under CC BY-NC. No commercial re-use. See rights and permissions. Published by BMJ.

Open Heart 2021;8:e001494corr1. doi:10.1136/openhrt-2020-001494corr1

(D) Check for updates 